\title{
Responses of simple optical standing wave sensors
}

\author{
Victor-Otto de Haan, ${ }^{1, *}$ Rudi Santbergen, ${ }^{2}$ Martijn Tijssen, ${ }^{2}$ and Miro Zeman ${ }^{2}$ \\ 'BonPhysics B.V., Laan van Heemstede 38, 3297 AJ Puttershoek, The Netherlands \\ ${ }^{2}$ Delft University of Technology, EWI Faculty, Mekelweg 4, 2628 CD Delft, The Netherlands \\ ${ }^{*}$ Corresponding author: victor@ bonphysics.nl
}

Received 23 January 2012; revised 13 March 2012; accepted 14 March 2012; posted 15 March 2012 (Doc. ID 162012); published 21 May 2012

\begin{abstract}
Optical standing wave sensors have been manufactured by amorphous silicon deposition. The responses of these sensors, when subjected to standing waves, have been calculated and measured. It is shown that the responses are different depending on the way the standing wave is created. The responses also depend on the thickness and material properties of the layers used to create the sensors. Quantitative agreement between measurements and model calculations can be obtained by including alignment errors, incoherent light interaction and scaling factors. The simple construction of the sensors allows for a broad application range. (C) 2012 Optical Society of America
\end{abstract}

OCIS codes: $\quad 280.4788,250.0040,230.5160,350.7420$.

\section{Introduction}

Optical standing waves were detected by Wiener [1], Ives [2] , and Jäger []ㅡ] by reflecting light to a surface. A standing wave sensor based on a thin absorbing layer in a vacuum photo-multiplier tube was constructed and successfully tested by Silvertooth [4]. A huge reduction in size was achieved by means of transparent thin photo diodes [5], [6]. These can be used for the construction of very small interferometers [7], [8]. In all these experiments the standing waves were created by passing the wave through the sensor and reflecting it back on a mirror through the sensor. This is denoted as Michelson-Morley geometry (see Fig. 1 left). Recently [9] it has been shown that the absorption of light in such sensors can be different when the standing waves are created by first splitting the beam and then redirecting the splitted beams so that they travel in opposite directions through the sensor. This is denoted as Sagnac geometry (see Fig. 1 right). In [9] the absorption was measured by a reduction of the transmitted intensity. Here, the response is measured by means of simple standing wave sensors based on amorphous silicon

$1559-128 \mathrm{X} / 12 / 163109-05 \$ 15.00 / 0$

(C) 2012 Optical Society of America deposition on a glass substrate. The sensors were constructed in such a way that a large part of the absorbed photons are converted into electrons. In the next section a description is given of the standing wave sensors and their fabrication method. Then, measurements for the two different situations are compared to theoretical calculations and the last two sections contain the discussion and conclusions.

\section{Standing Wave Sensors}

The sensors consist of a semitransparent hydrogenated amorphous silicon ( $a-\mathrm{Si}: \mathrm{H})$ absorber layer sandwiched between two transparent contact layers. The layers were deposited onto a $0.7 \mathrm{~mm}$ thick glass substrate (Corning Eagle XG). The transparent contact layers have a design thickness of $176 \mathrm{~nm}$ and consist of indium doped tin oxide (ITO) deposited using RF-sputter deposition. This thickness was chosen because model calculations showed that a layer thickness corresponding to a multiple of half a wavelength (in ITO) yielded the largest modulation of the absorption (The sensors will be operated at a wavelength of $632.8 \mathrm{~nm}$.). The composition of the sputtering target is $\mathrm{In}_{2} \mathrm{O}_{3}(90 \mathrm{wt} \%)-\mathrm{SnO}_{2}(10 \mathrm{wt} \%)$ with pure argon at a pressure of $10 \mu \mathrm{bar}$ as sputtering gas. The substrate temperature was kept at $150{ }^{\circ} \mathrm{C}$ and a power density of $1.25 \mathrm{~W} / \mathrm{cm}^{2}$ was used, resulting 

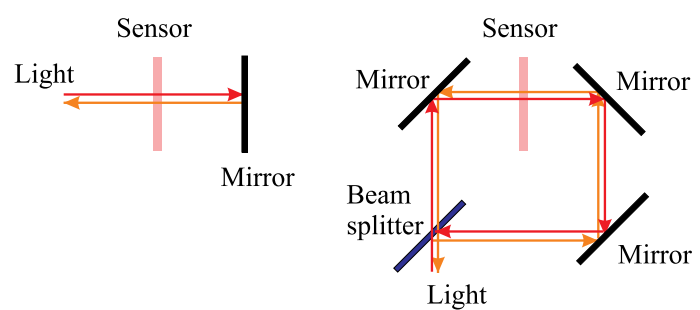

Fig. 1. (Color online) Michelson-Morley (left) and Sagnac (right) geometry for creating standing waves through a sensor.

in a deposition rate of $8 \mathrm{~nm} / \mathrm{min}$. The $a-\mathrm{Si}: \mathrm{H}$ layer was deposited using plasma enhanced chemical vapor deposition (PECVD), with a $\mathrm{SiH}_{4}$ flow of $40 \mathrm{sccm}$ and a pressure of $0.7 \mathrm{mbar}$. The substrate was kept at $180{ }^{\circ} \mathrm{C}$ and a power density of $24 \mathrm{~mW} / \mathrm{cm}^{2}$ was used, resulting in a deposition rate of $12 \mathrm{~nm} / \mathrm{min}$. Two sensors were fabricated with a design $a-\mathrm{Si}: \mathrm{H}$ layer thickness of $148 \mathrm{~nm}$ and $185 \mathrm{~nm}$ for sensors A and B. These thicknesses were chosen because a layer thickness corresponding to a multiple of half a wavelength (in $\alpha$-Si: $\mathrm{H}$ ) yielded the largest (and in between these values, the lowest) modulation in case of Sagnac geometry. For the Michelson-Morely geometry, the amplitude of the modulation varies much less with varying $s$-Si:H layer thickness. Note that only intrinsic $a$-Si:H was deposited and no doped layers are used. Because of nonuniformities during deposition the layer thicknesses of ITO and $a-\mathrm{Si}: \mathrm{H}$ may deviate up to $5 \%$ from the design values. Figure 2 shows the external quantum efficiency (EQE) of both sensors as a function of wavelength. The EQE indicates the probability that an incident photon generates a charge carrier that is collected at the contacts. A bias voltage of $-1 \mathrm{~V}$ was applied to make sure that all generated charge carriers are collected. For wavelengths larger than $500 \mathrm{~nm}$ the EQE of both sensors reduces with increasing wavelength. This is due to the decreasing absorption coefficient of the $a-\mathrm{Si}: \mathrm{H} \mathrm{ab}-$ sorber layer. For a wavelength of $632.8 \mathrm{~nm}$ the absorption coefficient of $a-\mathrm{Si}: \mathrm{H}$ is $2.0 \mu \mathrm{m}^{-1}$, implying that light will lose about $18 \%$ of its intensity due to absorption in $a$-Si:H for every $100 \mathrm{~nm}$ travelled.

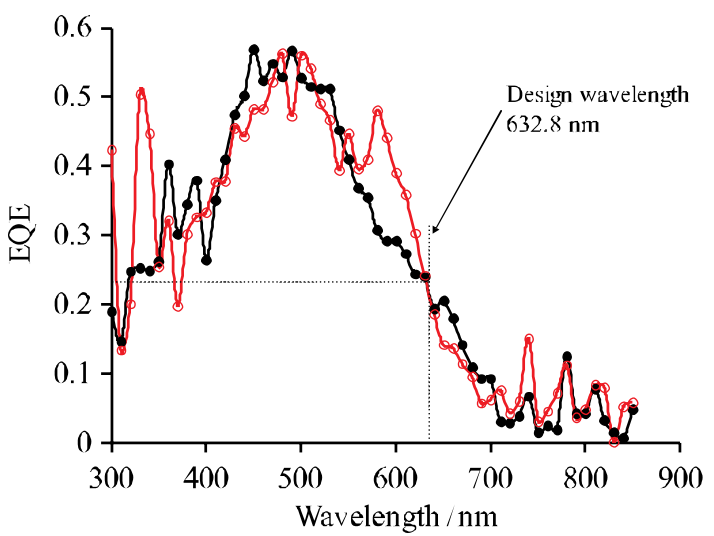

Fig. 2. (Color online) External quantum efficiency (EQE) of both sensors as a function of wavelength: black line/dots: A; red line/ circles: B.
Consequently, a sensor with a thickness of the order of $100 \mathrm{~nm}$ will absorb a sufficient amount of light to generate a measurable current and at the same time will be sufficiently transparent to be used as a standing wave detector. Compared to $a$-Si:H, the absorption coefficient of ITO is about 20 times lower at this wavelength and absorption losses in ITO will not play an important role.

\section{Experiments}

The sensors were tested by submitting them to standing waves created by the Michelson-Morley and Sagnac geometry (see Fig. 1).

\section{A. Michelson-Morley Geometry}

The Michelson-Morley geometry (see Fig. 3) was realized by inserting light of a $0.5 \mathrm{~mW}$ Coherent 200 , stabilized HeNe laser (stability $1 \mathrm{MHz}$, wavelength $632.8 \mathrm{~nm}$ ) into a SM600 optical fiber with a fiber collimator (PAF-X-5-B, Thorlabs, focal length $4.6 \mathrm{~mm}$, $\oslash 0.86 \mathrm{~mm}$ waist). The optical fiber was fed into another similar fiber collimator which focused the laser beam at the surface of a di-electric mirror (BB05E02, Thorlabs). The intensity coupled out of the fiber was determined by collecting all of it on a amplified silicon photo detector (PDA36A, Thorlabs) and was approximately $50 \mu \mathrm{W}$. The mirror was aligned so that the light was back reflected into the fiber collimator. The distance between the fiber collimator and the mirror was approximately $17 \mathrm{~cm}$. The standing wave sensor was mounted in the beam on a piezo crystal (AEO505D08F, Thorlabs, sensitivity $61 \pm 15 \mathrm{~nm} / \mathrm{V}$ ) for lateral displacement. It was aligned so that its surface was perpendicular to the beam with an accuracy of approximately $1 \mathrm{mrad}$. The alignment involved multiple reflections between sensor surface and mirror. These surfaces always reflect part of the light beam (both specular and diffuse). The diffuse reflection can be observed, indicating the position of the light beam. If these surfaces are not parallel, then at each reflection the direction of the beam is changed and hence the reflections do not coincide. The spot size location can be observed up to an accuracy of order $0.1 \mathrm{~mm}$ and the distance between the sensor and the mirror was approximately $5 \mathrm{~cm}$. Hence the alignment accuracy can be $0.1 /(50 \times 2)=$ $1 \mathrm{mrad}$. The limitation is the need of an observable change of the spot position and the limited distance between mirror and sensor. Further the alignment was checked by optimizing the modulation of the

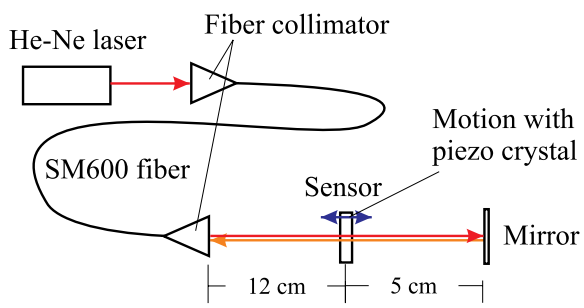

Fig. 3. (Color online) Michelson-Morley geometry for creating standing waves through a sensor. 


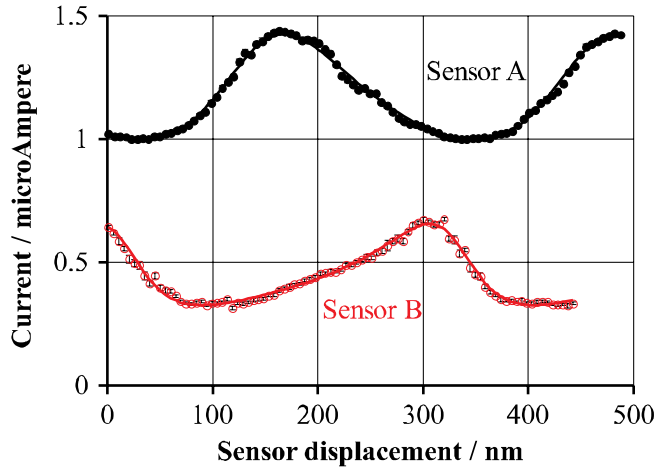

Fig. 4. (Color online) Responses of standing wave sensors placed in an optical standing wave created by the Michelson-Morley geometry (see Fig. 3) as function of sensor displacement. Black dots: sensor A, red circles: sensor B. The lines represent the fitted models. The estimated error bars have approximately the same size as the symbols.

sensor signal as the sensor was displaced by the piezo crystal. The current produced by the standing wave sensor was measured by means of a photo diode amplifier (PDA200C, Thorlabs) without bias voltage. The dark-current was dependent on the sensor used and subtracted from the signal. For the two sensors the signal as function of the sensor displacement is shown in Fig. 4.

\section{B. Sagnac Geometry}

The Sagnac geometry (see Fig. 5) was realized using the same laser, collimators, alignment procedure and photo current detection method. A SM600 optical fiber coupler (coupling ratio 50\%, FC632-50B Thorlabs) was used as beam splitter. The fiber coupler coherently splits the beam into two beams emerging via two fiber collimators in opposite directions. The fiber collimators were aligned so that the light from one fiber collimator was inserted into the other one. The distance between the sensor and the collimators was approximately 11 and $12 \mathrm{~cm}$. For the two sensors the signal as function of the sensor displacement is shown in Fig. $\underline{6}$.

\section{Discussion}

The lines in Figs. 4 and 6 represent model calculations with parameters shown in Table 1 . The model calculations are based on a completed solution of the

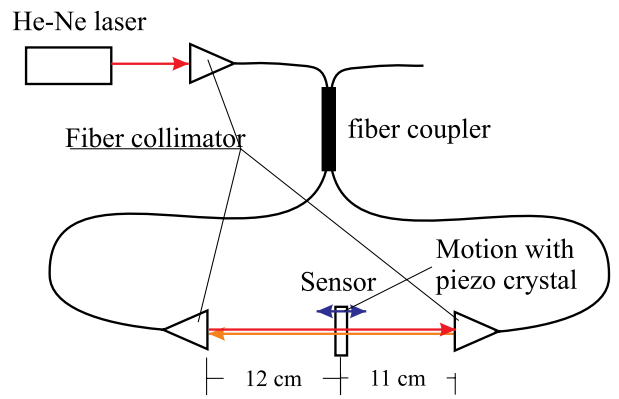

Fig. 5. (Color online) Sagnac geometry for creating standing waves through a sensor.

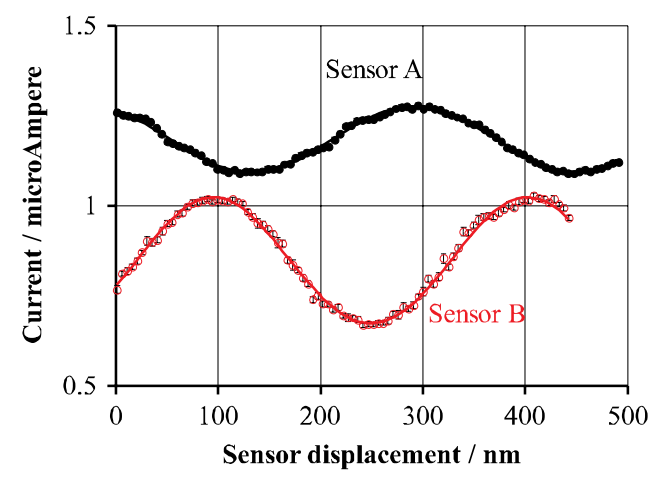

Fig. 6. (Color online) Responses of standing wave sensors placed in an optical standing wave created by the Sagnac geometry (see Fig. 5) as function of sensor displacement. Black dots: sensor A, red circles: sensor B. The lines represent the fitted models. The estimated error bars have approximately the same size as the symbols.

electromagnetic field created in the sensor by the interfering light waves and are described in detail in [9]. The model takes into account the full light path between sensor and mirror in case of MichelsonMorley geometry and between beam splitter and sensor in case of Sagnac geometry. This includes the multiple reflections between the mirror and the sensor and in the layers of the sensor. The refractive index of ITO and $a$-Si:H used in the model calculations was determined using separate reflectiontransmission measurements of the individual layers on glass. The agreement between calculations and experiment is very good. The difference in the sensor responses is due to the different thicknesses of the $a$ Si:H layers. To obtain such good fits, a scaling factor and offset needed to be introduced according to $i(x)=i_{o}+\hat{i} a(x)$, where $i(x)$ equals the measured photo current as function of sensor displacement, $i_{o}$ the current offset, $\hat{i}$ the scaling factor, and $a(x)$ the calculated relative absorption of the light in the $a$-Si:H layer as function of sensor displacement. The results are shown in Table 1 . The difference in piezo sensitivity is due to the fact that the sensors were mounted on different piezo crystals and is in the range quoted by the manufacturer. The offset can be explained as due to the part of the beam that did not create standing waves. This can be due to misalignment of the sensor in the beam or by misalignment of the beams directed in opposite direction. Another possibility is a different rotation of polarization inside the fibers. In these measurements this was not determined independently. The offset relative to the scaling factor was less for the MichelsonMorley geometry than for the Sagnac geometry indicating that this is less of a problem for the first. This can be explained by the fact that polarization rotation in air is negligible but in (twisted) fibers can be substantial [10].

The fitted thickness for the amorphous silicon of sensor B deviated by some $12 \%$ from the design value. This is somewhat larger than the expected deviation but still a reasonable value. One should keep in 
Table 1. Design and Fitted Model Parameters for the Optical Standing Wave Sensors

\begin{tabular}{|c|c|c|c|c|}
\hline & Sensor A & & Sensor B & \\
\hline Refractive index glass substrate & 1.50 & - & 1.50 & - \\
\hline Thickness glass substrate & 0.7 & $\mathrm{~mm}$ & 0.7 & $\mathrm{~mm}$ \\
\hline Refractive index ITO & $1.8+0.0055 i$ & - & $1.8+0.0055 i$ & - \\
\hline Refractive index $a-\mathrm{Si}: \mathrm{H}$ & $4.27+0.103 i$ & - & $4.27+0.103 i$ & - \\
\hline Design thickness $a$-Si:H & 148 & $\mathrm{~nm}$ & 185 & $\mathrm{~nm}$ \\
\hline Fitted thickness $a-\mathrm{Si}: \mathrm{H}$ & $149 \pm 1$ & $\mathrm{~nm}$ & $208 \pm 1$ & $\mathrm{~nm}$ \\
\hline EQE according to fit & $0.25 \pm 0.01$ & - & $0.28 \pm 0.01$ & - \\
\hline Fitted scale factor MM & $1.110 \pm 0.024$ & $\mu \mathrm{A} / 50 \mu \mathrm{W}$ & $0.888 \pm 0.015$ & $\mu \mathrm{A} / 50 \mu \mathrm{W}$ \\
\hline Fitted offset MM & $0.927 \pm 0.002$ & idem & $0.248 \pm 0.006$ & idem \\
\hline Fitted scale factor Sagnac & $0.212 \pm 0.020$ & idem & $0.387 \pm 0.004$ & idem \\
\hline Fitted offset Sagnac & $0.524 \pm 0.003$ & idem & $0.282 \pm 0.002$ & idem \\
\hline Fitted piezo sensitivity & $82.4 \pm 0.3$ & $\mathrm{~nm} / \mathrm{V}$ & $74.3 \pm 0.3$ & $\mathrm{~nm} / \mathrm{V}$ \\
\hline
\end{tabular}

mind that the calculations are sensitve to the optical thickness of the amorphous silicon layer. Hence, according to the calculations it would be possible that also the refractive index of this layer is different from the expected one. This would however also effect the calculations of the other sensor as they were manufactured from the same material. As the other sensor's fit results are close to the design values a change in refractive index seems unlikely.

The EQE (Fig. 2) is measured with a single beam passing through the sensor. It has a different wavelength dependence for both sensors and some peaks are clearly visible. This is due to the wavelength dependent interference from beams partly reflected at the layer interfaces in the sensor. This occurs in all absorbing sensors, but is more pronounced here because the layer thicknesses are of the same order as the wavelength of the light. The fact that the EQE at $632.8 \mathrm{~nm}$ is approximately the same for both sensors is a result of the specific interference pattern at this wavelength. As soon as the sensor is placed in a standing wave the light interference pattern in the sensor will also change, resulting in a change of absorption. If the EQE is calculated using the design values of the sensor parameters the results are 0.25 for sensor A and 0.16 for sensor B. According to Fig. 2, the measured values coincide at $0.23 \pm$ 0.01 . If the EQE is calculated with the fitted parameters, these values become $0.25 \pm 0.01$ and $0.28 \pm$ 0.01 , respectively. These match the measured values a bit better. A perfect match could be made by assuming that the absorption coefficient in the silicon is $20 \%$ lower. At $632.8 \mathrm{~nm}$ a shift in bandgap of the $a$-Si:H of just $-0.05 \mathrm{eV}$ could reduce the absorption coefficient by this amount. Such a shift could be caused by slightly different deposition conditions. This changes also the other fit parameters a bit, but they remain in the expected range. This gives confidence that the model is consistent.

Finally, the fitted scale factor varies for the different measurements between 0.2 and $1.1 \mu \mathrm{A} / 50 \mu \mathrm{W}$. Although the variation can be explained by means of adjustments of the optical set-up between the measurements, the average value is very small compared to the theoretical value of $25 \mu \mathrm{A} / 50 \mu \mathrm{W}$. The reason for this difference is not clear. It can be due to the very small bias applied for these measurements, resulting in incomplete extraction of the photo-generated charge carriers. Another possibility is a saturation effect due to the small cross section (order of $1 \mathrm{~mm}^{2}$ ) of the applied laser beam.

\section{Conclusions}

The measurements show that standing waves can easily be detected with simple sensors based on a thin layer of amorphous silicon. The responses of the sensors depend on the way the standing waves are created. The modulation of the measured response as function of the position of the sensor in the standing wave is large enough for detection even at a low beam intensity of the order of microwatts. The alignment of both beams to create the standing waves and the sensor in the beam is crucial. According to the fit results, the produced sensors agree reasonable to their design parameters indicating that the production technique is good. An advantage of the designed sensors is that they can easily be applied to any optical surface, turning such a surface into a simple and cheap solution for optical standing wave detection in applications ranging from distance measurements to Fourier spectrometry.

\section{References}

1. O. Wiener, "Stehende lichtwellen und die schwingungsrichtung polarisirten lichtes," Ann. Phys. Chemie 276, 203-243 (1890).

2. H. E. Ives and T. C. Fry, "Standing light waves; repetition of an experiment by Wiener, using a photoelectric probe surface," J. Opt. Soc. Am. 23, 73-83 (1933).

3. H. Jäger, "Ein neues beobachtumgsverfahrem für stehende lichtwellen," Ann. Phys. 5, 280-296 (1939).

4. E. W. Silvertooth and S. F. Jacobs, "Standing wave sensor," Appl. Opt. 22, 1274-1275 (1983).

5. L. Carraresi, E. A. De Souza, D. A. B. Miller, W. Y. Jan, and J. E. Cunningham, "Wavelength-selective detector based on a quantum well in a standing wave," Appl. Phys. Lett. 64, 134-137 (1994). 
6. M. Sasaki, X. Mi, and K. Hane, "Standing wave detection and interferometer application using a photodiode thinner than optical wavelength," Appl. Phys. Lett. 75, 2008-2010 (1999).

7. H-J. Büchner, H. Stiebig, V. Mandryka, E. Bunte, and G. Jäger, "An optical standing-wave interferometer for displacement measurements," Meas. Sci. Technol. 14, 311 (2003).

8. Y. Li, X. Mi, M. Sasaki, and K. Hane, "Precision optical displacement sensor based on ultra-thin film photodiode type optical interferometers," Meas. Sci. Technol. 14, 479-483 (2003).

9. V. O. de Haan de, R. Santbergen, M. Tijssen, and M. Zeman, "Standing waves in fiber optic interferometers," Appl. Opt. 50, 5674-5687 (2011).

10. R. Ulrich, S. C. Rashleigh, and W. Eickhoff, "Bending-induced birefringence in single-mode fibers," Opt. Lett. 5, 273-275 (1980). 